\title{
Towards Rational Designing of Efficient Sensitizers Based on Thiophene and Infrared Dyes for Dye-Sensitized Solar Cells
}

\author{
Ahmad Irfan, ${ }^{1}$ Abdullah G. Al-Sehemi, ${ }^{1,2,3}$ and Shabbir Muhammad ${ }^{4}$ \\ ${ }^{1}$ Department of Chemistry, Faculty of Science, King Khalid University, P.O. Box 9004, Abha 61413, Saudi Arabia \\ ${ }^{2}$ Unit of Science and technology, Faculty of Science, King Khalid University, P.O. Box 9004, Abha 61413, Saudi Arabia \\ ${ }^{3}$ Center of Excellence for Advanced Materials Research, King Khalid University, Abha 61413, Saudi Arabia \\ ${ }^{4}$ Department of Physics, Faculty of Science, King Khalid University, P.O. Box 9004, Abha 61413, Saudi Arabia
}

Correspondence should be addressed to Ahmad Irfan; irfaahmad@gmail.com

Received 7 July 2013; Revised 8 September 2013; Accepted 23 September 2013; Published 2 January 2014

Academic Editor: Aijun Du

Copyright (C) 2014 Ahmad Irfan et al. This is an open access article distributed under the Creative Commons Attribution License, which permits unrestricted use, distribution, and reproduction in any medium, provided the original work is properly cited.

Geometries, electronic properties, and absorption spectra of the dyes which are a combination of thiophene based dye (THPD) and IR dyes (covering IR region; TIRBD1-TIRBD3) were performed using density functional theory (DFT) and time dependent density functional theory (TD-DFT), respectively. Different electron donating groups, electron withdrawing groups, and IR dyes have been substituted on THPD to enhance the efficiency. The bond lengths of new designed dyes are almost the same. The lowest unoccupied molecular orbital energies of designed dyes are above the conduction band of $\mathrm{TiO}_{2}$ and the highest occupied molecular orbital energies are below the redox couple revealing that TIRBD1-TIRBD3 would be better sensitizers for dye-sensitized solar cells. The broad spectra and low energy gap also showed that designed materials would be efficient sensitizers.

\section{Introduction}

There exist many potential renewable energy technologies in the form of solid-state devices, such as, solar cells, which convert solar energy in the form of light to the more practical form of electricity. In 1991, Regan and Gratzel invented dyesensitized solar cells (DSSCs). Solar cells are among the most important devices that can be used to solve the world's energy and environmental needs. The DSSCs are still in the early stages. The DSSC is a promising solar cell technology [14]. The metal-free organic dyes as sensitizers such as borondipyrromethene (BODIPY) dyes [5], indoline dyes (D102 and D149) [6], infrared absorbing thiapenta-carbocyanine dye [7] and so forth, have been intensively studied. Improvement of absorption of the solar spectrum has been investigated mainly by adding different types of dopants such as transition metal elements, nonmetal elements, nitrogen, sulfur, boron, carbon nanotubes, and so forth [8-16].

The geometric characteristics of organic thiophene based dye (THPD) are of great attention for many applications, including photodynamic therapy light harvesting, nonlinear absorption, and optical storage. The UV/visible absorption areas of organic thiophene based dye are of great value when preparing them as sensitizers for solar cells. Theoretical investigations of the physical properties of the dye sensitizers are very important in order to disclose the relationship among the performance, structure, and properties. It is also supportive to design and produce novel dye sensitizer with high performance. Recently, we have designed the THPD and then explained it as a good sensitizer (17a). Generally THPD absorbs in the visible region. We are expecting that the combination of THPD and IR dye might enhance the efficiency of DSSCs due to shifting of the absorption spectra toward longer wavelength.

In the present study we present a theoretical effort intended at giving an enhanced thoughtful role on the geometric and electronic properties and absorption spectra. We have used different electron donating, electron withdrawing groups, and IR dyes to enhance the absorption toward longer wavelength. The IR dyes have been substituted with 


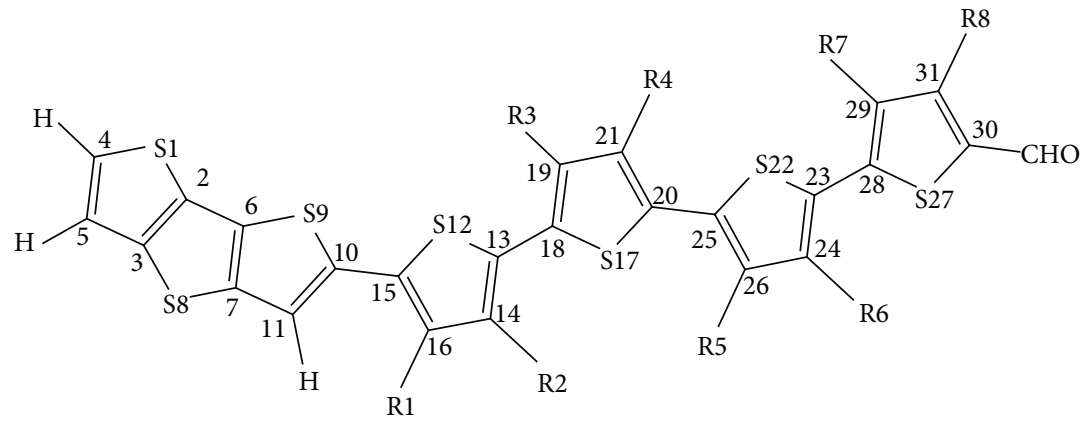

(a)

\begin{tabular}{ll}
\hline Derivatives & Positions \\
\hline THPD & $\mathrm{CN}$ at R1, R4; nitro at R2, R3; methoxy at R7, R6; t-butyl group at R5, R8 \\
TIRBD1 & $\mathrm{CN}$ at R6, R7; nitro at R5; methoxy at R1, R4, and IR dye at R8 \\
TIRBD2 & $\mathrm{CN}$ at R6, R4; nitro at R5, R7; methoxy at R1; IR dye at R8 \\
TIRBD3 & $\begin{array}{l}\mathrm{CN} \text { at R6, R7; } \mathrm{CH}_{3} \text { at R1; ethyl at R3; nitro at R5; methoxy at R2, R4; IR } \\
\text { dye at R8 }\end{array}$ \\
\hline
\end{tabular}

(b)

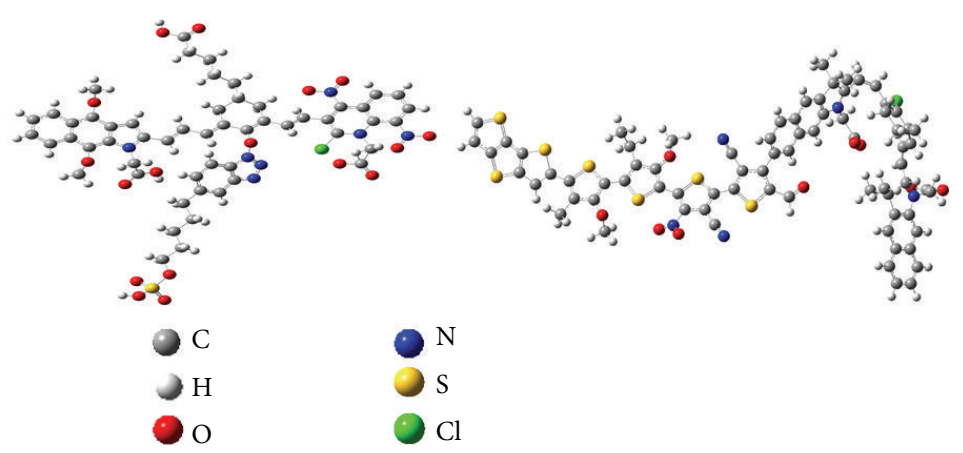

(c)

FIGURE 1: The new designed dyes investigated in the present study (right bottom is optimized geometry of TIRBD3 at B3LYP/6-31G** level of theory).

conjugated chain with the thiophene unit to enhance the absorption spectra toward longer wavelength. The structureproperty relationship has been discussed.

\section{Computational Details}

In our previous study it has been pointed out that B3LYP/6$31 \mathrm{G}^{* *}$ level of theory is a good choice for the systems having thiophene units $[17,18]$. The TD-B3LYP/6-31G ${ }^{* *}$ was used previously as a good choice of method to compute the absorption spectra which were in good agreement with the experimental data $[17,18]$. Thus, geometries of new designed thiophene and IR based dyes (TIRBD1 and TIRB2) see Figure 1 have been optimized using density functional theory (DFT) at B3LYP/6-31G ${ }^{* *}$ level of theory. We have computed absorption spectra at TD-B3LYP/6-31G ${ }^{* *}$ level of theory $[17,18]$. All the calculations have been performed by using Gaussian 09 [19]. The absorption spectra graphs have been obtained by using GaussSum [20].

\section{Results and Discussions}

3.1. Geometries. In this section we have compared the geometrical parameters with computed data of THPD which has been previously designed and demonstrated as a good sensitizer [18]. In the TIRBD1, C15-C16 has been decreased by $0.01 \AA$, because cyanide group at $\mathrm{R} 1$ has been replaced by methoxy group which is an electron donating group. The C13-C14 and C18-C19 have been increased by 0.018 and $0.020 \AA$. The C25-C26 bond length has been increased by $0.012 \AA$ because methoxy group at R 5 has been replaced with nitro group which is a strong electron withdrawing group that attracts all the charge density towards itself, due to which bond length stretches. The C30-C31 increased by $0.01 \AA$ 
because alkyl group has been replaced with IR dye at R8 position which is in conjugation with the system and attracts all the charge density towards itself resulting in bond length stretches.

In TIRBD2, C13-C14 has been increased by $0.012 \AA$. The C28-C29 bond length has been increased by $0.010 \AA$, because methoxy group has been replaced with nitro group which is a strong electron withdrawing group that attracts all the charge density towards itself, due to which bond length stretches. The C13-C14, C18-S17, C20-S17, C18-C19, S22-C25, C23-S22, C28-C29, and C31-C29 of TIRBD1 are 0.006, 0.009, 0.011, $0.015,0.02,0.011,0.015$, and $0.010 \AA$ larger than TIRBD2. The C20-C25, C24-C26, C23-C28 of TIRBD1 are shorter 0.008, 0.007 , and $0.011 \AA$ compared to TIRBD2. Both of the dyes have no significant change in bond lengths and bond angles compared to each other; see Tables 1 and 2.

In the TIRBD3, C15-C16 has been decreased by $0.01 \AA$ because cyanide group at $\mathrm{R} 5$ has been replaced by methoxy group which is electron donating. $\mathrm{C} 13-\mathrm{C} 14$ and $\mathrm{C} 18-\mathrm{C} 19$ have been increased by 0.015 and $0.013 \AA$. By introducing the IR dye in place of alkyl group C23-C24, bond length increased by $0.01 \AA$.

In TIRBD1, C15-S12-C13 and C18-S17-C20 bond angles decrease by $0.54^{\circ}$ and $0.67^{\circ}$, respectively. The C23-S22-C25 and C30-S27-C28 increase by $1.83^{\circ}$ and $1.11^{\circ}$, respectively. InTIRBD2, C15-S12-C13 decreases by $0.96^{\circ}$, while the $\mathrm{C} 18-$ S17-C20, C23-S22-C25, and C30-S27-C28 increase by $0.01^{\circ}$, $0.84^{\circ}$, and $0.69^{\circ}$, respectively. In TIRBD2, the C15-S12-C13 and C18-S17-C20 decrease by $0.94^{\circ}$ and $0.47^{\circ}$, while C23S22-C25 and C30-S27-C28 increase by $0.72^{\circ}$ and $1.39^{\circ}$, respectively.

\subsection{Electronic Structure and Absorption Spectra. The HOMO} and LUMO are shown by green and blue lines, respectively. The region between green and blue lines shows the HOMOLUMO energy gap of system. The red line shows the density of states (DOS) spectrum; see Figure 2.

The HOMO and LUMO energies of THPD are $-6.32 \mathrm{eV}$ and $-2.96 \mathrm{eV}$, which lead to HOMO-LUMO energy gap $3.36 \mathrm{eV}$. In TIRBD1, HOMO and LUMO show the energy of $-4.90 \mathrm{eV}$ and $-3.02 \mathrm{eV}$, respectively. The HOMO-LUMO energy gap of the TIRBD1 is $1.88 \mathrm{eV}$. In TIRBD2, HOMO and LUMO show the energy of $-5.13 \mathrm{eV}$ and $-3.20 \mathrm{eV}$, respectively. The LUMO-HOMO energy gap of the TIRBD2 is $1.93 \mathrm{eV}$. In TIRBD3, HOMO and LUMO energies are $-5.23 \mathrm{eV}$ and $-3.40 \mathrm{eV}$, respectively. The LUMO-HOMO energy gap of the TIRBD2 is $1.83 \mathrm{eV}$. The HOMO-LUMO energy gap of investigated dyes is smaller than the THPD and BDT $(3.18 \mathrm{eV})$. The sequence in $\mathrm{HOMO}$ energies from higher to lower is TIRBD1 > TIRBD2 > TIRBD3 > THPD, while the LUMO energies are THPD > TIRBD1 > TIRBD2 > TIRBD3. The trend in the HOMO-LUMO energy gap has been observed as THPD > TIRBD2 > TIRBD1 > TIRBD3.

The basic function is to prepare such dyes which are very much competent for DSSC which fulfill the basic requirements of design. Firstly, the sensitizing dyes must firmly bond to the photocatalyst plane to make sure there is very rapid electron insertion into the conduction band
TABLE 1: The bond lengths (BL) of TIRBD1-TIRBD3 in $\AA$ at $\mathrm{B} 3 \mathrm{LYP} / 6-31 \mathrm{G}^{* *}$ level of theory.

\begin{tabular}{lcccc}
\hline BL & THPD & TIRBD1 & TIRBD2 & TIRBD3 \\
\hline S1-C2 & 1.726 & 1.726 & 1.726 & 1.726 \\
C4-C5 & 1.365 & 1.365 & 1.365 & 1.365 \\
C3-C5 & 1.419 & 1.419 & 1.419 & 1.419 \\
C2-C3 & 1.393 & 1.393 & 1.393 & 1.393 \\
C2-C6 & 1.413 & 1.413 & 1.413 & 1.413 \\
C6-C7 & 1.394 & 1.394 & 1.394 & 1.394 \\
S1-C4 & 1.734 & 1.734 & 1.734 & 1.734 \\
S8-C7 & 1.744 & 1.744 & 1.744 & 1.744 \\
S8-C3 & 1.742 & 1.742 & 1.742 & 1.742 \\
C1-C7 & 1.408 & 1.408 & 1.408 & 1.408 \\
C11-C10 & 1.381 & 1.381 & 1.381 & 1.381 \\
S9-C10 & 1.760 & 1.760 & 1.760 & 1.760 \\
S9-C6 & 1.721 & 1.721 & 1.721 & 1.721 \\
C10-C15 & 1.440 & 1.444 & 1.442 & 1.442 \\
C15-C16 & 1.391 & 1.381 & 1.384 & 1.381 \\
C15-S12 & 1.735 & 1.737 & 1.732 & 1.732 \\
C13-S12 & 1.725 & 1.741 & 1.743 & 1.747 \\
C13-C14 & 1.371 & 1.389 & 1.383 & 1.386 \\
C14-C16 & 1.429 & 1.420 & 1.423 & 1.423 \\
C13-C18 & 1.460 & 1.443 & 1.444 & 1.444 \\
C18-S17 & 1.723 & 1.743 & 1.734 & 1.727 \\
C20-S17 & 1.739 & 1.740 & 1.729 & 1.737 \\
C19-C21 & 1.430 & 1.413 & 1.414 & 1.437 \\
C18-C19 & 1.374 & 1.394 & 1.379 & 1.387 \\
C21-C20 & 1.390 & 1.396 & 1.395 & 1.387 \\
C20-C25 & 1.443 & 1.436 & 1.444 & 1.464 \\
S22-C25 & 1.727 & 1.748 & 1.728 & 1.732 \\
C23-S22 & 1.734 & 1.734 & 1.723 & 1.723 \\
C23-C24 & 1.385 & 1.384 & 1.380 & 1.395 \\
C25-C26 & 1.385 & 1.397 & 1.393 & 1.380 \\
C24-C26 & 1.435 & 1.426 & 1.433 & 1.430 \\
C23-C28 & 1.474 & 1.447 & 1.458 & 1.446 \\
C28-S27 & 1.749 & 1.718 & 1.715 & 1.726 \\
S27-C30 & 1.691 & 1.730 & 1.730 & 1.727 \\
C28-C29 & 1.381 & 1.399 & 1.384 & 1.370 \\
C31-C29 & 1.460 & 1.430 & 1.420 & 1.398 \\
\hline & & & & \\
\hline
\end{tabular}

of $\mathrm{TiO}_{2}$. Secondly, the LUMO of the dye must be passably higher than the conduction band of $\mathrm{TiO}_{2}$ for efficient charge insertion, and the HOMO of the dye must be lower than the hole-transport material (HTM) for efficient regeneration of the oxidized dye. To design efficient sensitizers for use in dye-sensitized solar cells, the following factor needs to be considered: a narrow band gap, with LUMO lying just above the conduction band and HOMO below the redox couple. As a model for nanocrystallinity the HOMO and LUMO energies of bare cluster $\left(\mathrm{TiO}_{2}\right)_{38}$ are -7.23 and $-4.1 \mathrm{eV}$, respectively, resulting in a HOMO-LUMO gap of $3.13 \mathrm{eV}$ [21]. Usually an energy gap of more than $0.2 \mathrm{eV}$ between the LUMO of the dye and the conduction band of the $\mathrm{TiO}_{2}$ is 
TABLE 2: Calculated bond angles in degree of investigated systems at B3LYP/6-31G** level of theory.

\begin{tabular}{lcccccccc}
\hline Bond angles & TIRBD1 & TIRBD2 & TIRBD3 & Bond angles & TIRBD1 & TIRBD2 & TIRBD3 & THPD \\
\hline C2-S1-C4 & 90.97 & 90.96 & 91.00 & C18-S17-C20 & 92.40 & 90.96 & 92.60 \\
S1-C4-C5 & 113.33 & 113.34 & 113.27 & S17-C18-C19 & 111.97 & 113.34 & 109.33 & 114.16 \\
S1-C2-C3 & 111.01 & 111.00 & 110.97 & S17-C20-C21 & 109.10 & 111.00 & 112.73 \\
C3-C5-C4 & 111.01 & 111.07 & 111.12 & C19-C21-C20 & 115.77 & 111.07 & 109.42 & 111.12 \\
C7-S8-C3 & 90.29 & 90.30 & 90.35 & C18-C19-C21 & 110.75 & 90.30 & 115.87 \\
S8-C3-C2 & 112.57 & 112.58 & 112.48 & C23-S22-C25 & 93.99 & 112.58 & 92.88 \\
S1-C2-C6 & 136.70 & 136.73 & 136.69 & S22-C23-C24 & 110.61 & 136.73 & 109.64 & 113.36 \\
S8-C7-C6 & 112.48 & 112.43 & 112.39 & S22-C25-C26 & 108.05 & 112.43 & 111.89 \\
S8-C7-C11 & 133.63 & 133.56 & 133.55 & C23-C24-C26 & 112.27 & 133.56 & 114.44 \\
C7-C6-S9 & 111.15 & 111.12 & 111.07 & C24-C26-C25 & 115.18 & 111.12 & 112.84 \\
C6-S9-C10 & 91.30 & 91.20 & 91.26 & C28-S27-C30 & 92.80 & 91.20 & 93.08 \\
C11-C10-S9 & 111.75 & 111.99 & 111.90 & S27-C28-C29 & 109.93 & 111.99 & 110.40 \\
C7-C11-C10 & 111.90 & 111.67 & 111.72 & S27-C30-C31 & 112.22 & 111.67 & 110.18 \\
C15-S12-C13 & 92.86 & 92.44 & 92.46 & C28-C29-C31 & 114.30 & 92.44 & 111.90 \\
S12-C15-C16 & 111.61 & 109.79 & 110.11 & C29-C31-C30 & 110.76 & 109.79 & 111.83 \\
\hline
\end{tabular}

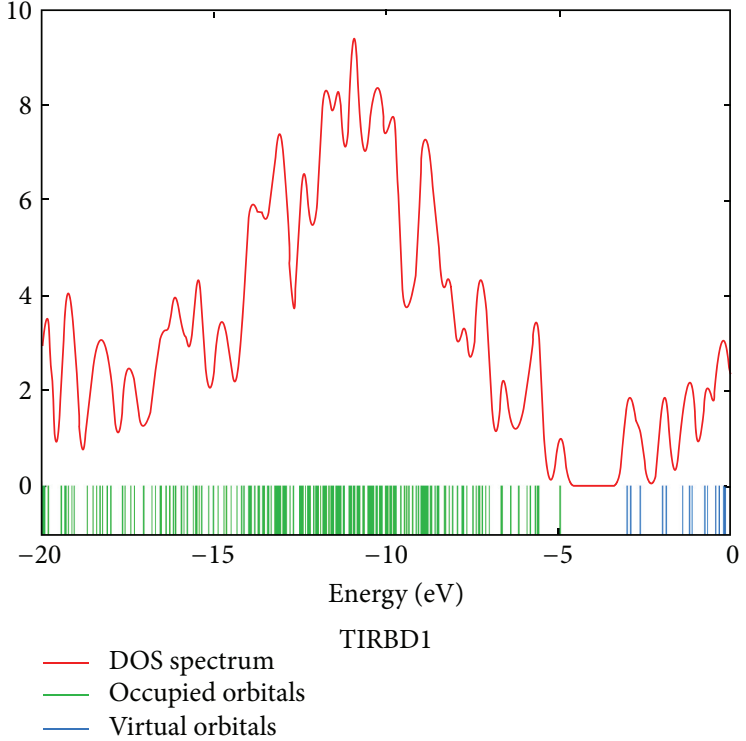

(a)

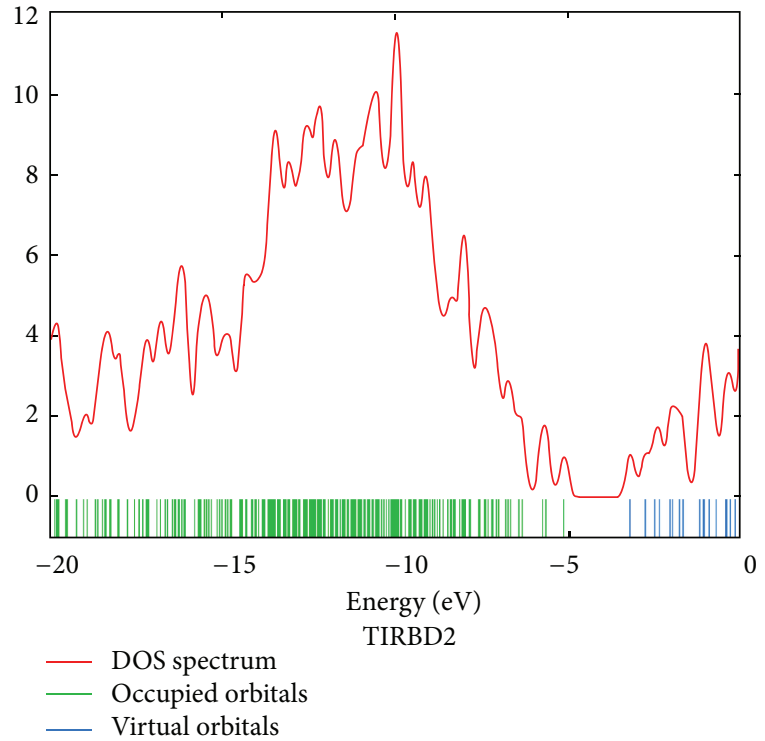

(b)

Figure 2: The DOS spectra at B3LYP/6-31G ${ }^{* *}$ level of theory.

necessary for effective electron injection [22]. The LUMO energies of TIRBD1-TIRBD3 are above the conduction band of $\mathrm{TiO}_{2}$. The HOMO of the redox couple $\left(\mathrm{I}^{-} / \mathrm{I}_{3}{ }^{-}\right)$is $-4.8 \mathrm{eV}$ [23]. It can be found that HOMOs of the designed dyes are below the redox couple. The smaller HOMO-LUMO energy gaps of TIRBD1-TIRBD3 showed that these materials would be efficient dyes as sensitizers. Here we discussed the distribution pattern of the HOMO and LUMO of TIRBD3 and found that the HOMO is distributed on IR dye, while LUMO is delocalized at THPD; see Figure 3. The charge distributed at electron withdrawing groups would also be anchoring groups revealing that electron would be transferable from anchoring group to metal oxide $\left(\mathrm{TiO}_{2}\right)$.
By substituting the IR dye at position R8 of THPD, the significant decrease in HOMO-LUMO energy gap has been observed in TIRBD1. We have changed the positions of electron donating and electron withdrawing groups. It is expected that the effect of electron donating and electron accepting groups is not so influential to increase or decrease the HOMO energies, LUMO energies or HOMO-LUMO energy gaps. In TIRBD1 and TIRBD2, the IR dyes are the same, but the positions and numbers of electron donating and electron accepting groups are different, while the significant effect to decrease the HOMO energies, LUMO energies, and HOMO-LUMO energy gap in studied sensitizers is expected due to the IR dyes. The smaller HOMO-LUMO energy 


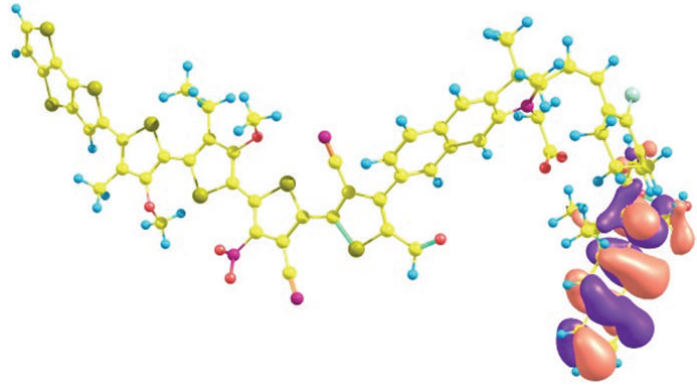

(a)

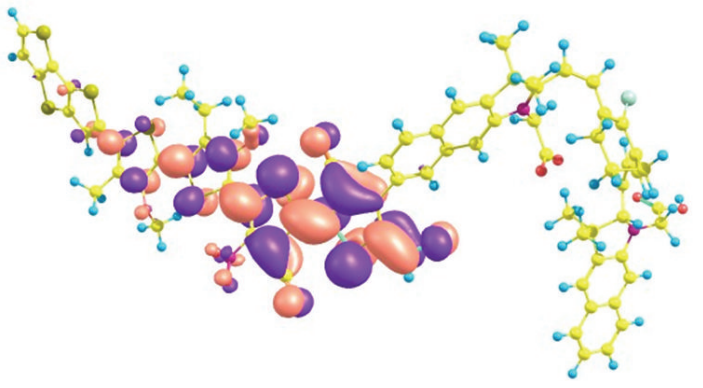

(b)

FIgURE 3: The distribution pattern of the HOMO and LUMO of representative system TIRBD3.

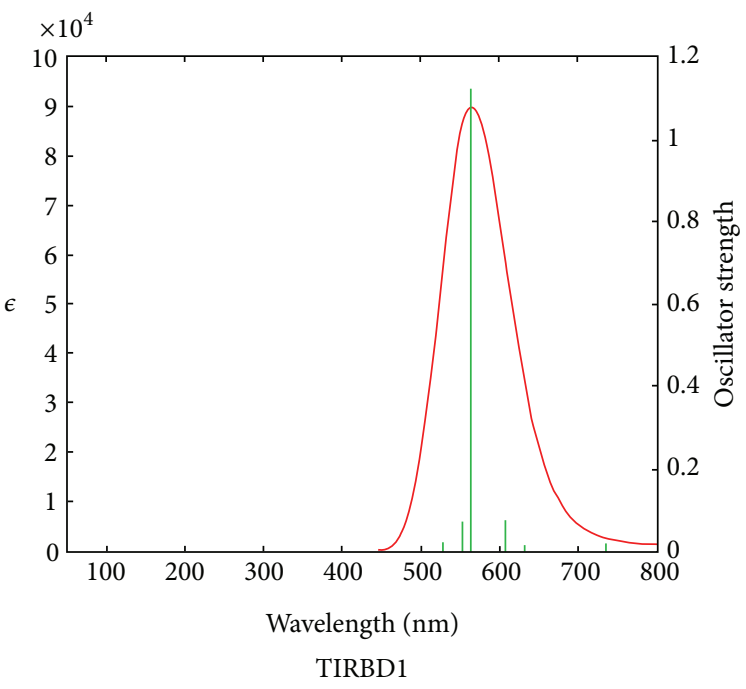

(a)

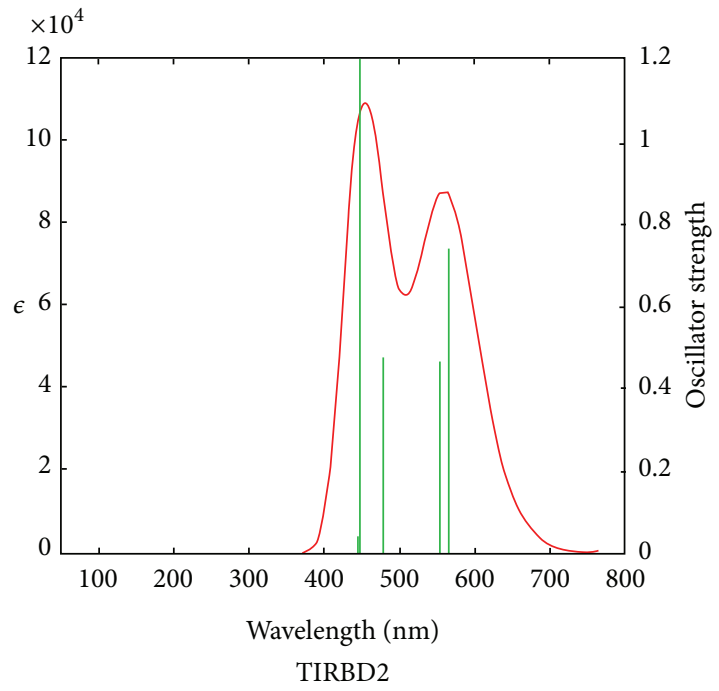

(b)

FIgURE 4: The absorption spectra of TIRBD1 and TIRBD2 at B3LYP/6-31G ${ }^{* *}$ level of theory.

gaps of TIRBD1-TIRBD3 than THPD are revealing that the introduction of IR dyes in the systems would be more efficient to reduce the HOMO-LUMO energy gap enhancing the efficiency as sensitizers.

The THPD (thiophene based) has been derived from BDT. Recently, we have optimized the geometry of BDT with solvent (chloroform according to experimental setup) and without solvent. Then, computed the absorption spectra by using PCM and CPCM as well as without solvent at two different levels of theories (B3LYP/6-31G ${ }^{* *}$ and PBE0/6-31G ${ }^{* *}$ ). The computed absorption spectra at B3LYP/6-31G ${ }^{* *}$ level of theory are more reliable than $\mathrm{PBE} 0 / 6-31 \mathrm{G}^{* *}$ level of theory. The computed absorption spectrum without solvent was observed as $414 \mathrm{~nm}$ which was in good agreement with experimental evidence: $440 \mathrm{~nm}$ (detail can be found in reference 18). Thus, in the present study, we have computed parameters without solvent to minimize the cost. Our previous study revealed that THPD showed two absorption peaks at $384 \mathrm{~nm}$ and $443 \mathrm{~nm}$, but the second peak is not prominent [18]. The absorption graph of the TIRBD1 demonstrated maximum absorption at $564 \mathrm{~nm}$. Absorption graph of the TIRBD2 illustrated the first peak at $447 \mathrm{~nm}$ and second peak at $571 \mathrm{~nm}$; see Table 3. The TIRBD2 would be better sensitizer having broad spectra as well $(400-600 \mathrm{~nm})$; see Figure 4. It was considered that thiophene core attached with IR dye would enhance the absorption spectra. Our investigation showed that TIRBD1 is $180 \mathrm{~nm}$ red shifted compared to THPD. On the other hand, $63 \mathrm{~nm}$ and $128 \mathrm{~nm}$ red shift in first and second peak has been observed in TIRBD2, respectively, compared to THPD. We were expecting that absorption spectra would be near IR due to the substitution of IR dye. As we have substituted the IR dye with thiophene core by conjugated chain, the absorption spectra shifted to blue shift might be due to the conjugated chain. Now, work on IR dyes and a thiophene core attached to each other with unconjugated carbon chain is under consideration. We expect that it would enhance the absorption spectra toward red shift near IR region.

\section{Conclusions}

The theoretical analysis showed that by substituting the thiophene with different electron withdrawing, electron donating groups, and IR dyes, the HOMO-LUMO energy gap reduces 
TABle 3: The HOMO energies $\left(E_{\mathrm{HOMO}}\right)$, LUMO energies $\left(E_{\mathrm{LUMO}}\right)$, HOMO-LUMO energy gap $\left(E_{\text {gap }}\right)$ in $\mathrm{eV}$, and absorption spectra (B and $\mathrm{Q}$ bands in $\mathrm{nm}$ ) of TIRBD1 and TIRBD2 at B3LYP/6-31G ${ }^{* *}$ and TD-B3LYP/6-31G** levels of theory, respectively.

\begin{tabular}{lccccc}
\hline Systems & $E_{\text {HOMO }}$ & $E_{\text {LUMO }}$ & $E_{\text {gap }}$ & B bands & Q bands \\
\hline THPD & -6.32 & -2.96 & 3.36 & 384 & 443 \\
TIRBD1 & -4.90 & -3.02 & 1.88 & - & 564 \\
TIRBD2 & -5.13 & -3.20 & 1.93 & 447 & 570 \\
TIRBD3 & -5.23 & -3.40 & 1.83 & & \\
\hline
\end{tabular}

and absorption range becomes broader. The LUMO energies of TIRBD1-TIRBD3 are above the conduction band of $\mathrm{TiO}_{2}$. The HOMOs of the designed dyes are below the redox couple and the smaller HOMO-LUMO energy gaps showed that these materials would be efficient dyes as sensitizers. The smaller HOMO-LUMO energy gaps of TIRBD1-TIRBD3 than THPD are revealing that the introduction of IR dyes in the systems would be more efficient to reduce the HOMO-LUMO energy gap and would be better sensitizers. This study would be helpful to design highly efficient light harvesting materials for DSSC.

\section{Conflict of Interests}

The authors declare that there is no conflict of interests regarding the publication of the paper.

\section{Acknowledgment}

The support and facilities provided by King Khalid University are gratefully acknowledged.

\section{References}

[1] B. O. Regan and M. Gratzel, "A low-cost, high-efficiency solar cell based on dye-sensitized colloidal $\mathrm{TiO}_{2}$ films," Nature, vol. 353, pp. 737-740, 1991.

[2] A. Hagfeldt and M. Grätzel, "Molecular photovoltaics," Accounts of Chemical Research, vol. 33, no. 5, pp. 269-277, 2000.

[3] M. Grätzel, "Solar energy conversion by dye-sensitized photovoltaic cells," Inorganic Chemistry, vol. 44, no. 20, pp. 6841-6851, 2005.

[4] C. Wadia, A. P. Alivisatos, and D. M. Kammen, "Materials availability expands the opportunity for large-scale photovoltaics deployment," Environmental Science and Technology, vol. 43, no. 6, pp. 2072-2077, 2009.

[5] X. Zhang, Y. Xiao, and X. Qian, "Highly efficient energy transfer in the light harvesting system composed of three kinds of boron-dipyrromethene derivatives," Organic Letters, vol. 10, no. 1, pp. 29-32, 2008.

[6] M. Pastore and F. De Angelis, "Aggregation of organic dyes on $\mathrm{TiO}_{2}$ in dye-sensitized solar cells models: an Ab initio investigation," ACS Nano, vol. 4, no. 1, pp. 556-562, 2010.

[7] M. T. Spitler and B. A. Parkinson, "Dye sensitization of single crystal semiconductor electrodes," Accounts of Chemical Research, vol. 42, no. 12, pp. 2017-2029, 2009.
[8] J. J. Yun, H. S. Jung, S. H. Kim, V. Vaithianathan, S. A. Jenekhe, and E. M. Han, "Chlorophyll-layer-inserted poly(3hexyl-thiophene) solar cell having a high light-to-current conversion efficiency up to 1.48\%," Applied Physics Letters, vol. 87, Article ID 123102, 2005.

[9] S. Klosek and D. Raftery, "Visible light driven V-doped $\mathrm{TiO}_{2}$ photocatalyst and its photooxidation of ethanol," Journal of Physical Chemistry B, vol. 105, no. 14, pp. 2815-2819, 2002.

[10] H. Haick and Y. Paz, "Long-range effects of noble metals on the photocatalytic properties of titanium dioxide," Journal of Physical Chemistry B, vol. 107, no. 10, pp. 2319-2326, 2003.

[11] J.-J. Yun, J. Peet, N.-S. Cho, G. C. Bazan, S. J. Lee, and M. Moskovits, "Insight into the Raman shifts and optical absorption changes upon annealing polymer/fullerene solar cells," Applied Physics Letters, vol. 92, no. 25, Article ID 251912, 2008.

[12] I. Gur, N. A. Fromer, M. L. Geier, and A. P. Alivisatos, "Materials science: air-stable all-inorganic nanocrystal solar cells processed from solution," Science, vol. 310 , no. 5747 , pp. $462-$ 465, 2005.

[13] D. J. Norris, A. L. Efros, and S. C. Erwin, "Doped nanocrystals," Science, vol. 319, no. 5871, pp. 1776-1779, 2008.

[14] X. Chen and C. Burda, "The electronic origin of the visible-light absorption properties of $\mathrm{C}$-, $\mathrm{N}$ - and $\mathrm{S}$-doped $\mathrm{TiO}_{2}$ nanomaterials," Journal of the American Chemical Society, vol. 130, no. 15, pp. 5018-5019, 2008.

[15] H. Li, X. Zhang, Y. Huo, and J. Zhu, "Supercritical preparation of a highly active S-doped $\mathrm{TiO}_{2}$ photocatalyst for methylene blue mineralization," Environmental Science and Technology, vol. 41, no. 12, pp. 4410-4414, 2007.

[16] S. In, A. Orlov, R. Berg et al., "Effective visible light-activated $\mathrm{B}$-doped and $\mathrm{B}, \mathrm{N}$-codoped $\mathrm{TiO}_{2}$ photocatalysts," Journal of the American Chemical Society, vol. 129, no. 45, pp. 13790-13791, 2007.

[17] A. Irfan, H. Aftab, and A. G. Al-Sehemi, "Push-pull effect on the geometries, electronic and optical properties of thiophene based dye-sensitized solar cell materials," Journal of Saudi Chemical Society, 2011.

[18] A. Irfan, M. Nadeem, M. Athar, F. Kanwal, and J. Zhang, "Electronic, optical and charge transfer properties of $\alpha, \alpha^{\prime}$-bis(dithieno[3,2-b:2' $3^{\prime}$-d] thiophene) (BDT) and its heteroatom-substituted analogues," Computational and Theoretical Chemistry, vol. 968, no. 1-3, pp. 8-11, 2011.

[19] M. J. Frisch, G. W. Trucks, H. B. Schlegel et al., Gaussian 09, Revision A.1, Gaussian, Inc., Wallingford, Conn, USA, 2009.

[20] N. M. O’Boyle, A. L. Tenderholt, and K. M. Langner, “Cclib: a library for package-independent computational chemistry algorithms," Journal of Computational Chemistry, vol. 29, no. 5, pp. 839-845, 2008.

[21] F. D. Angelis, S. Fntacci, and A. Selloni, "Alignment of the dye's molecular levels with the $\mathrm{TiO}_{2}$ band edges in dye-sensitized solar cells: a DFT-TDDFT study ", Nanotechnology, vol. 19, Article ID 424002, 8 pages, 2008.

[22] S. Ito, S. M. Zakeeruddin, R. Humphry-Baker et al., "High-efficiency organic-dye-sensitized solar cells controlled by nanocrystalline- $\mathrm{TiO}_{2}$ electrode thickness," Advanced Materials, vol. 18, no. 9, pp. 1202-1205, 2006.

[23] M. P. Balanay and D. H. Kim, "DFT/TD-DFT molecular design of porphyrin analogues for use in dye-sensitized solar cells," Physical Chemistry Chemical Physics, vol. 10, no. 33, pp. 51215127, 2008. 

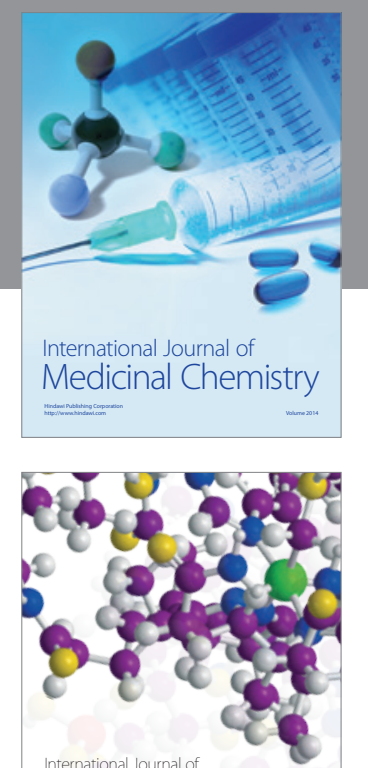

\section{Carbohydrate} Chemistry

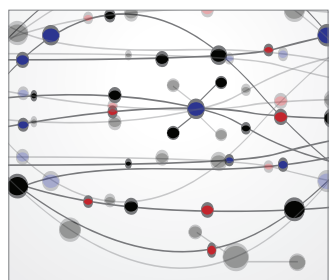

The Scientific World Journal
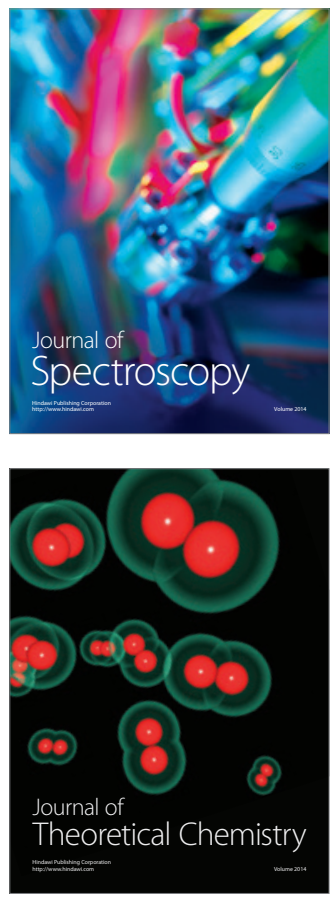
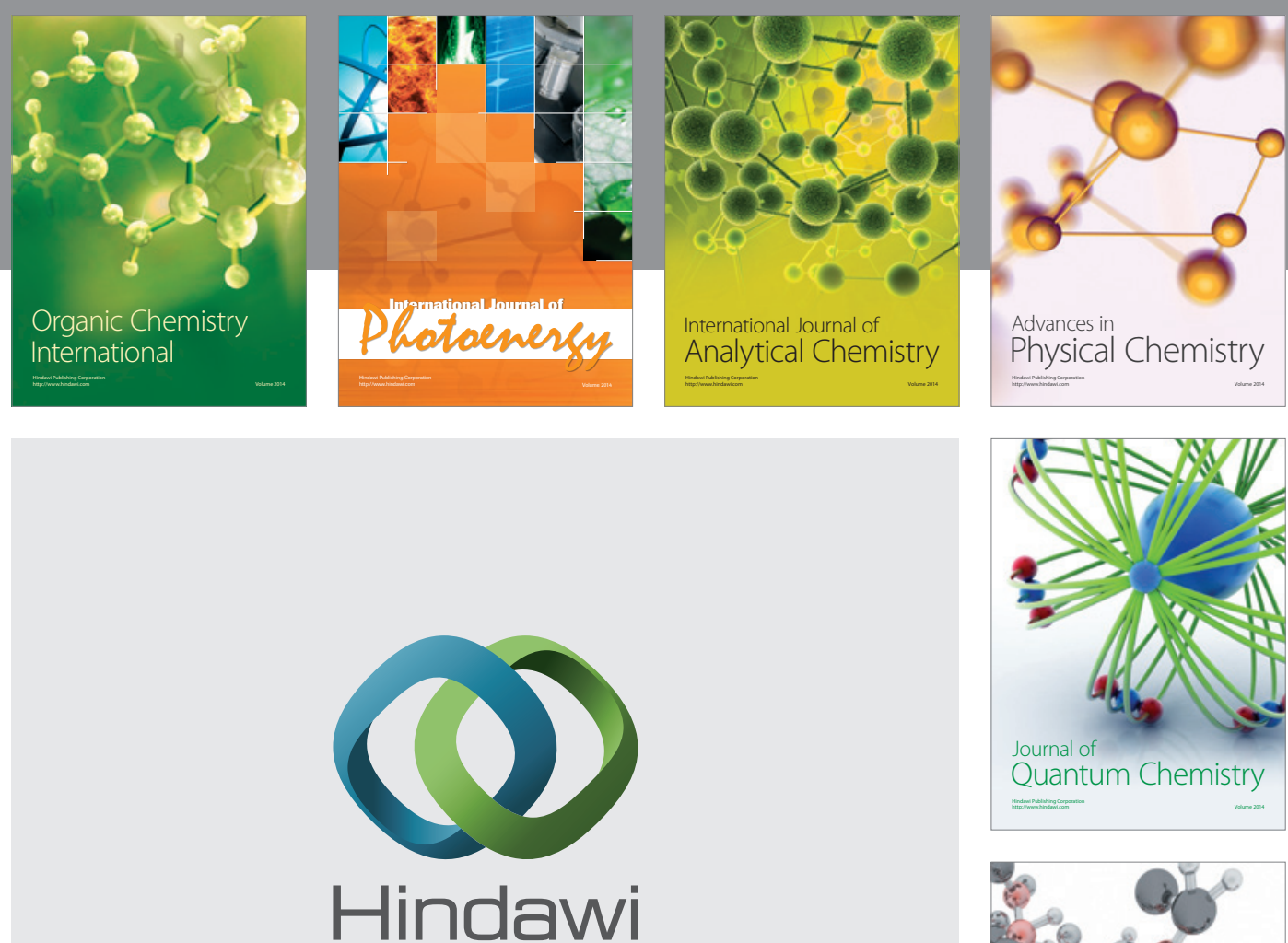

Submit your manuscripts at

http://www.hindawi.com

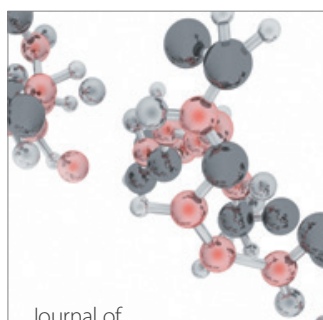

Analytical Methods

in Chemistry

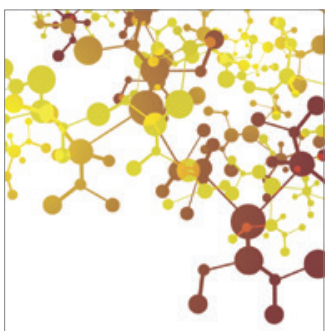

Journal of

Applied Chemistry

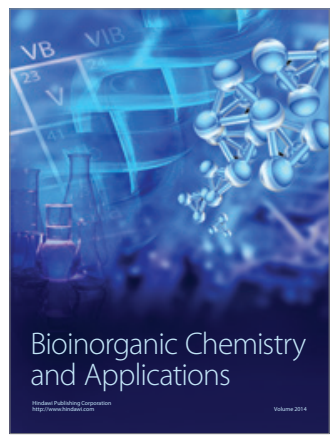

Inorganic Chemistry
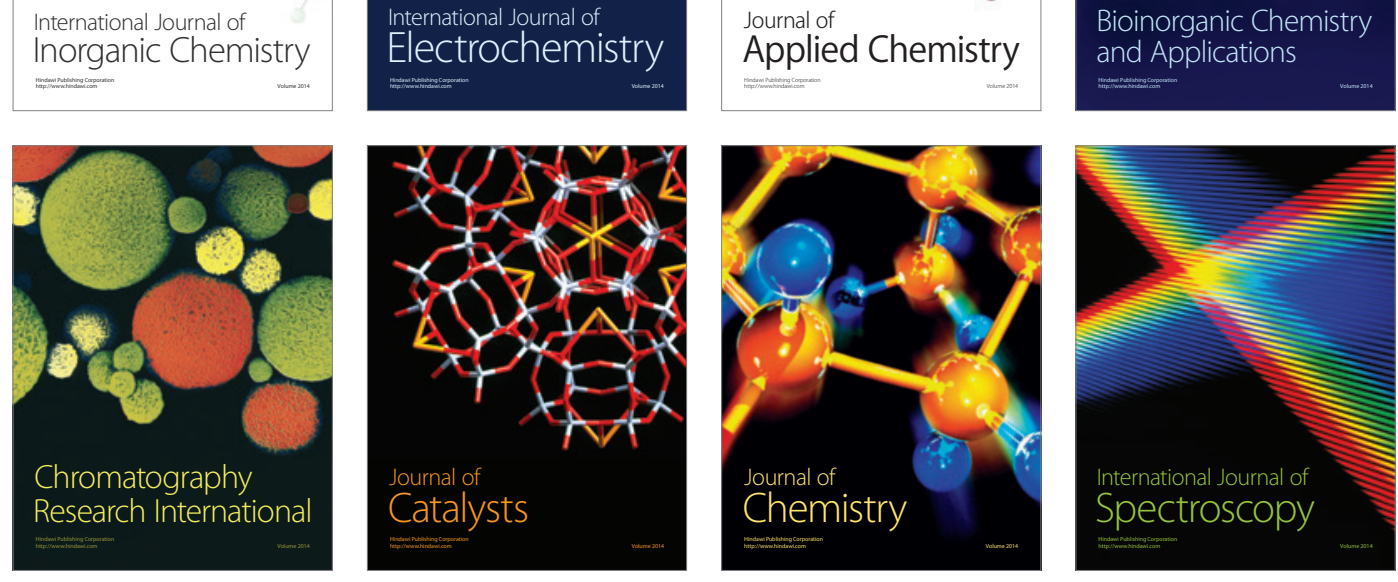